\title{
On the infimum of quantum effects
}

\author{
Aurelian Gheondea ${ }^{\text {a) }}$ \\ Department of Mathematics, Bilkent University, 06800 Bilkent, Ankara, Turkey and \\ Institutul de Matematică al Academiei Române, C.P. 1-764, 014700 București, România \\ Stanley Gudder ${ }^{\text {b) }}$ \\ Department of Mathematics, University of Denver, Denver, Colorado 80208 \\ Peter Jonas ${ }^{\mathrm{c})}$ \\ Institut für Mathematik, Technische Universität Berlin, 10623 Berlin, Germany
}

(Received 24 September 2004; accepted 21 March 2005; published online 12 May 2005)

The quantum effects for a physical system can be described by the set $\mathcal{E}(\mathcal{H})$ of positive operators on a complex Hilbert space $\mathcal{H}$ that are bounded above by the identity operator. While a general effect may be unsharp, the collection of sharp effects is described by the set of orthogonal projections $\mathcal{P}(\mathcal{H}) \subseteq \mathcal{E}(\mathcal{H})$. Under the natural order, $\mathcal{E}(\mathcal{H})$ becomes a partially ordered set that is not a lattice if $\operatorname{dim} \mathcal{H}$ $\geqslant 2$. A physically significant and useful characterization of the pairs $A, B \in \mathcal{E}(\mathcal{H})$ such that the infimum $A \wedge B$ exists is called the infimum problem. We show that $A \wedge P$ exists for all $A \in \mathcal{E}(\mathcal{H}), P \in \mathcal{P}(\mathcal{H})$ and give an explicit expression for $A \wedge P$. We also give a characterization of when $A \wedge(I-A)$ exists in terms of the location of the spectrum of $A$. We present a counterexample which shows that a recent conjecture concerning the infimum problem is false. Finally, we compare our results with the work of Ando on the infimum problem. (C) 2005 American Institute of Physics. [DOI: 10.1063/1.1904704]

\section{INTRODUCTION}

A quantum mechanical measurement with just two values 1 and 0 (or yes and no) is called a quantum effect. These elementary measurements play an important role in the foundations of quantum mechanics and quantum measurement theory. ${ }^{3-5,7,14,16,18}$ We shall follow the Hilbert space model for quantum mechanics in which effects are represented by positive operators on a complex Hilbert space $\mathcal{H}$ that are bounded above by the identity operator $I$. In this way the set of effects $\mathcal{E}(\mathcal{H})$ becomes

$$
\mathcal{E}(\mathcal{H})=\{A \in \mathcal{B}(\mathcal{H}): 0 \leqslant A \leqslant I\} .
$$

The set of orthogonal projections $\mathcal{P}(\mathcal{H}) \subseteq \mathcal{E}(\mathcal{H})$ corresponds to sharp effects while a general $A$ $\in \mathcal{E}(\mathcal{H})$ may be unsharp (fuzzy, imprecise). Employing the usual order $A \leqslant B$ for the set of bounded self-adjoint operators $\mathcal{S}(\mathcal{H})$ on $\mathcal{H}$, we see that $(\mathcal{E}(\mathcal{H}), \leqslant)$ is a partially ordered set. It is well known that $(\mathcal{E}(\mathcal{H}), \leqslant)$ is not a lattice if $\operatorname{dim} \mathcal{H} \geqslant 2$. However, if the infimum $A \wedge B$ of $A, B$ $\in \mathcal{E}(\mathcal{H})$ exists then $A \wedge B$ has the important property of being the largest effect that physically implies both $A$ and $B$. It would thus be of interest to give a physically significant and useful characterization of when $A \wedge B$ exists. This so-called infimum problem has been considered for at least 10 years. ${ }^{2,10-12,17,19}$

Before discussing the progress that has been made toward solving the infimum problem, let us compare the situation with that of the partially ordered set $(\mathcal{S}(\mathcal{H}), \leqslant)$. Of course, if $A, B$

\footnotetext{
${ }^{a)}$ Electronic mail: aurelian@fen.bilkent.edu.tr and gheondea@imar.ro

${ }^{b}$ Electronic mail: sgudder@math.du.edu

${ }^{c)}$ Electronic mail: jonas@ @ath.tu-berlin.de
} 
$\in \mathcal{S}(\mathcal{H})$ are comparable, that is, $A \leqslant B$ or $B \leqslant A$, then $A \wedge B$ exists and is the smaller of the two. A surprising result of Kadison ${ }^{15}$ states that the converse holds. Thus, for $A, B \in \mathcal{S}(\mathcal{H}), A \wedge B$ exists in $\mathcal{S}(\mathcal{H})$ if and only if $A$ and $B$ are comparable. We conclude that $(\mathcal{S}(\mathcal{H}), \leqslant)$ is an antilattice which is as far from being a lattice as possible. The situation is quite different in $(\mathcal{E}(\mathcal{H}), \leqslant)$. In fact it is well known that $P \wedge Q$ exists in $\mathcal{E}(\mathcal{H})$ for all $P, Q \in \mathcal{P}(\mathcal{H})$. More generally, we shall show that $A \wedge P$ exists in $\mathcal{E}(\mathcal{H})$ for all $A \in \mathcal{E}(\mathcal{H}), P \in \mathcal{P}(\mathcal{H})$ and give an explicit expression for $A \wedge P$. The existence of $A \wedge P$ has already been proved in Ref. 18 but we present a different proof here.

For $A, B \in \mathcal{E}(\mathcal{H})$ let $P_{A, B}$ be the orthogonal projection onto the closure of $\operatorname{Ran}\left(A^{1 / 2}\right) \cap \operatorname{Ran}\left(B^{1 / 2}\right)$. It is shown in Ref. 19 that if $\operatorname{dim} \mathcal{H}<\infty$ then $A \wedge B$ exists in $\mathcal{E}(\mathcal{H})$ if and only if $A \wedge P_{A, B}$ and $B \wedge P_{A, B}$ are comparable and in this case $A \wedge B$ is the smaller of the two. This was considered to be a solution to the infimum problem for the case $\operatorname{dim} \mathcal{H}<\infty$ and it was conjectured in Ref. 19 that this result also holds in general. One of our main results is that this conjecture is false. We shall present an example of a pair $A, B \in \mathcal{E}(\mathcal{H})$ with $\operatorname{dim} \mathcal{H}=\infty$ for which $A \wedge B$ exists in $\mathcal{E}(\mathcal{H})$ but $A \wedge P_{A, B}$ and $B \wedge P_{A, B}$ are not comparable. In addition, we prove that, assuming $A \wedge B$ exists, $P_{A, B}$ is the smallest of all orthogonal projections $P$ having the property that $(A \wedge P) \wedge(B \wedge P)$ exists and $(A \wedge P) \wedge(B \wedge P)=A \wedge B$. Combined with the counter-example as described before, this means that, in the infinite dimensional case, there is no orthogonal projection to replace $P_{A, B}$ and have a positive solution to the infimum problem.

The negation $A^{\prime}$ of an effect $A$ is defined to be the effect $A^{\prime}=I-A$. Physically, $A^{\prime}$ is the effect $A$ with its values 1 and 0 reversed. We also present a simple spectral characterization of when $A \wedge A^{\prime}$ exists in $\mathcal{E}(\mathcal{H})$. The result is essentially the same as Theorem 2 in Ref. 2, with the difference that we express the condition in terms of the location of the spectrum of $A$ and the proof is based on the matrix representations obtained in the preceding section.

Ando has given a solution to the infimum problem in terms of a generalized shorted operator. ${ }^{2}$ However, in our opinion, these shorted operators do not have a physical significance in contrast to the operationally defined operators $A \wedge P_{A, B}$ and $B \wedge P_{A, B}$. Finally, we discuss the relationship between our work and that of Ando. First, we show that the shorted operator of $A$ by $B$ is always smaller than $A \wedge P_{A, B}$. Actually, it is the fact, that in the infinite dimensional case, the shorted operator of $A$ by $B$ can be strictly smaller than $A \wedge P_{A, B}$, that is responsible for the failure of a solution of the infimum problem similar to the finite dimensional case. This can be viewed from the counter-example as before, but we record also a simpler one that illustrates this situation.

We now briefly discuss connections between the infimum problem and physics. Quantum effects have been studied by mathematicians and physicists for over 40 years. ${ }^{5,16,17}$ Besides the applications of effect-valued measures in quantum measurement theory, many researchers consider effects to be the basic elements of important quantum structures. In recent times quantum effects have been organized into a structure called an effect algebra ${ }^{7,10}$ and their order properties have been studied. ${ }^{11,12,17}$ Among other things, the effect algebra $\mathcal{E}(\mathcal{H})$ is a partially ordered set and if $A \wedge B$ exists for $A, B, \in \mathcal{E}(\mathcal{H})$, then this effect has important physical properties. In particular, among all the effects that have a smaller probability of occurring than both $A$ and $B, A \wedge B$ has the largest probability. Thus if $A \wedge B$ exists, then $A \wedge B$ has a crucial physical significance. In the case where $A$ and $B$ are sharp, $A$ and $B$ are projections, $A \wedge B$ always exists and is the projection onto the intersection of their ranges. But if $A$ and $B$ are not sharp, the situation is much more complicated. An interesting special case is when $A \in \mathcal{E}(\mathcal{H})$ and $P \in \mathcal{P}(\mathcal{H})$. In this case $A \wedge P$ always exists and if $A$ and $P$ commute (are compatible) then $A \wedge P=A P$. However, if $A$ and $P$ do not commute an explicit closed form expression for $A \wedge P$ has been difficult to obtain and is now presented in Theorem 2.2. We can now define conditional probabilities

$$
\operatorname{prob}(A \mid P)=\operatorname{prob}(A \wedge P) / \operatorname{prob}(P)
$$

and conditional measurements and these may have useful physical applications. Finally, our Example 4.2 gives a surprising phenomenon that does not occur in finite dimensional Hilbert spaces. The existence of effects such as those in this example may have interesting physical significance. 


\section{INFIMUM OF A QUANTUM EFFECT AND A SHARP EFFECT}

We first record a parametrization of bounded positive $2 \times 2$ matrices with operator entries, in terms of operator balls.

In the following we make use of the Frobenius-Schur factorization: for $T, X, Y, Z$ bounded operators on appropriate spaces and $T$ boundedly invertible, we have

$$
\left[\begin{array}{cc}
T & X \\
Y & Z
\end{array}\right]=\left[\begin{array}{cc}
I & 0 \\
Y T^{-1} & I
\end{array}\right]\left[\begin{array}{cc}
T & 0 \\
0 & Z-Y T^{-1} X
\end{array}\right]\left[\begin{array}{cc}
I & T^{-1} X \\
0 & I
\end{array}\right]
$$

For instance, by using Frobenius-Schur factorizations and a perturbation argument one can obtain the following classical result of Shmulyan. ${ }^{21}$

Theorem 2.1: Let $A \in \mathcal{B}(\mathcal{H})$ be self-adjoint and $\mathcal{H}=\mathcal{H}_{1} \oplus \mathcal{H}_{2}$ an orthogonal decomposition of $\mathcal{H}$. Then $A \geqslant 0$ if and only if it has a matrix representation of the following form:

$$
A=\left[\begin{array}{cc}
A_{1} & A_{1}^{1 / 2} \Gamma A_{2}^{1 / 2} \\
A_{2}^{1 / 2} \Gamma^{*} A_{1}^{1 / 2} & A_{2}
\end{array}\right] \text { with respect to } \mathcal{H}=\mathcal{H}_{1} \oplus \mathcal{H}_{2},
$$

where $A_{1} \in \mathcal{B}\left(\mathcal{H}_{1}\right)^{+}, A_{2} \in \mathcal{B}\left(\mathcal{H}_{2}\right)^{+}$, and $\Gamma \in \mathcal{B}\left(\mathcal{H}_{2}, \mathcal{H}_{1}\right)$ is contractive.

In addition, the operator $\Gamma$ can be chosen in such a way that $\operatorname{Ker}(\Gamma) \supseteq \operatorname{Ker}\left(A_{2}\right)$ and $\operatorname{Ker}\left(\Gamma^{*}\right) \supseteq \operatorname{Ker}\left(A_{1}\right)$, and in this case it is unique.

For two effects $A, B \in \mathcal{E}(\mathcal{H})$ we denote by $A \wedge B$, the infimum, equivalently, the greatest lower bound, of $A$ and $B$ over the partially ordered set $(\mathcal{E}(\mathcal{H}), \leqslant)$, if it exists. To be more precise, $A \wedge B$ is an operator in $\mathcal{E}(\mathcal{H})$ uniquely determined by the following properties: $A \wedge B \leqslant A, A \wedge B \leqslant B$, and an arbitrary operator $D \in \mathcal{E}(\mathcal{H})$ satisfies both $D \leqslant A$ and $D \leqslant B$ if and only if $D \leqslant A \wedge B$. Characterizations of the existence of infimum for positive operators have been obtained for the finitedimensional case in Ref. 19, and in general in Ref. 2.

In Theorem 4.4 of Ref. 19 it is proved that the infimum $A \wedge P$ exists for any $A \in \mathcal{E}(\mathcal{H})$ and $P \in \mathcal{P}(\mathcal{H})$. As a consequence of Theorem 2.1 we can obtain an explicit description of $A \wedge P$, together with another proof of the existence.

Theorem 2.2: For any $A \in \mathcal{E}(\mathcal{H})$ and $P \in \mathcal{P}(\mathcal{H})$ the infimum $A \wedge P$ exists, more precisely, if $A$ has the matrix representation as in (2.2) with respect to the orthogonal decomposition $\mathcal{H}$ $=\operatorname{Ran}(P) \oplus \operatorname{Ker}(P)$, where $A_{1} \in \mathcal{E}(\operatorname{Ran}(P)), A_{2} \in \mathcal{E}(\operatorname{Ker}(P))$, and $\Gamma \in \mathcal{B}(\operatorname{Ker}(P)$, $\operatorname{Ran}(P))$, with $\|\Gamma\| \leqslant 1, \operatorname{Ker}(\Gamma) \supseteq \operatorname{Ker}\left(A_{2}\right)$ and $\operatorname{Ker}\left(\Gamma^{*}\right) \supseteq \operatorname{Ker}\left(A_{1}\right)$, then

$$
A \wedge P=\left[\begin{array}{cc}
A_{1}^{1 / 2}\left(I-\Gamma \Gamma^{*}\right) A_{1}^{1 / 2} & 0 \\
0 & 0
\end{array}\right] \quad \text { with respect to } \mathcal{H}=\operatorname{Ran}(P) \oplus \operatorname{Ker}(P) .
$$

Proof: Let $A \in \mathcal{E}(\mathcal{H})$ and $P \in \mathcal{P}(\mathcal{H})$. In the following we consider the orthogonal decomposition $\mathcal{H}=\operatorname{Ran}(P) \oplus \operatorname{Ker}(P)$. By Theorem $2.1 A$ has a matrix representation as in (2.2), with $A_{1}$ $\in \mathcal{B}(\operatorname{Ran}(P))^{+}, A_{2} \in \mathcal{B}(\operatorname{Ker}(P))^{+}$, and $\Gamma \in \mathcal{B}(\operatorname{Ker}(P), \operatorname{Ran}(P))$, with $\|\Gamma\| \leqslant 1, \operatorname{Ker}(\Gamma) \supseteq \operatorname{Ker}\left(A_{2}\right)$ and $\operatorname{Ker}\left(\Gamma^{*}\right) \supseteq \operatorname{Ker}\left(A_{1}\right)$. Since $A \leqslant I$ it follows that $A_{1} \leqslant I_{\operatorname{Ran}(P)}$ and $A_{2} \leqslant I_{\operatorname{Ker}(P)}$. Consider the operator $D \in \mathcal{B}(\mathcal{H})$, defined by the matrix in (2.3). Clearly $0 \leqslant D \leqslant P$, in particular $D \in \mathcal{E}(\mathcal{H})$. In addition,

$$
A-D=\left[\begin{array}{cc}
A_{1}^{1 / 2} \Gamma \Gamma^{*} A_{1}^{1 / 2} & A_{1}^{1 / 2} \Gamma A_{2}^{1 / 2} \\
A_{2}^{1 / 2} \Gamma^{*} A_{1}^{1 / 2} & A_{2}
\end{array}\right]=\left[\Gamma^{*} A_{1}^{1 / 2} A_{2}^{1 / 2}\right]^{*}\left[\Gamma^{*} A_{1}^{1 / 2} A_{2}^{1 / 2}\right] \geqslant 0,
$$

hence $A \geqslant D$.

Let $C \in \mathcal{E}(\mathcal{H})$ be such that $C \leqslant A, P$. From $C \leqslant P$ it follows that $C P=P C=C$ and hence

$$
C=\left[\begin{array}{cc}
C_{1} & 0 \\
0 & 0
\end{array}\right] \text { with respect to } \mathcal{H}=\operatorname{Ran}(P) \oplus \operatorname{Ker}(P) .
$$

Then 


$$
0 \leqslant A-C=\left[\begin{array}{cc}
A_{1}-C_{1} & A_{1}^{1 / 2} \Gamma A_{2}^{1 / 2} \\
A_{2}^{1 / 2} \Gamma^{*} A_{1}^{1 / 2} & A_{2}
\end{array}\right] .
$$

The matrix with operator entries in (2.4) can be factored as

$$
\left[\begin{array}{cc}
I_{\operatorname{Ran}(P)} & 0 \\
0 & A_{2}^{1 / 2}
\end{array}\right]\left[\begin{array}{cc}
A_{1}-C_{1} & A_{1}^{1 / 2} \Gamma \\
\Gamma^{*} A_{1}^{1 / 2} & I_{\operatorname{Ker}(P)}
\end{array}\right]\left[\begin{array}{cc}
I_{\operatorname{Ran}(P)} & 0 \\
0 & A_{2}^{1 / 2}
\end{array}\right] .
$$

Note that by $\operatorname{Ker}(\Gamma) \supseteq \operatorname{Ker}\left(A_{2}\right)$ or, equivalently, $\overline{\operatorname{Ran}\left(\Gamma^{*}\right)} \subseteq \overline{\operatorname{Ran}\left(A_{2}\right)}, A-C$ and each of the factors of (2.5) map the subspace $\mathcal{H}^{\prime}=\operatorname{Ran}(P) \oplus \overline{\operatorname{Ran}\left(A_{2}\right)}$ into itself. Since $\operatorname{diag}\left(I_{\operatorname{Ran}(P)} A_{2}^{1 / 2}\right)$ regarded as an operator on $\mathcal{H}^{\prime}$, is symmetric and has dense range, $A-C \geqslant 0$ implies that the middle term in (2.5) regarded as an operator in $\mathcal{H}^{\prime}$ is non-negative. By performing a Frobenius-Schur factorization of this middle term, we find $A_{1}^{1 / 2} \Gamma \Gamma^{*} A_{1}^{1 / 2} \leqslant A_{1}-C_{1}$, that is, $C_{1} \leqslant A_{1}^{1 / 2}\left(I_{\operatorname{Ran}(P)}-\Gamma \Gamma^{*}\right) A_{1}^{1 / 2}$, or, equivalently, $C \leqslant D$.

We thus proved that $A \wedge P$ exists and has the matrix representation as in (2.3).

Remark 2.3: If $A \in \mathcal{E}(\mathcal{H}), E_{A}$ is the spectral function of $A$ and $\Delta$ is a Borel subset of $[0,1]$, then $A \wedge E_{A}(\Delta)=A E_{A}(\Delta)$. This is an immediate consequence of Theorem 2.2. The second to last sentence in the proof of Theorem 2.2 can also be demonstrated by using the well-known fact that any operator matrix of the form

$$
\left[\begin{array}{ll}
A & B \\
B^{*} & I
\end{array}\right]
$$

is positive if and only if $A \geqslant 0$ and $B B^{*} \leqslant A$.

Let $A, B \in \mathcal{E}(\mathcal{H})$. By $P_{A, B}$ we denote the orthogonal projection onto the closure of $\operatorname{Ran}\left(A^{1 / 2}\right) \cap \operatorname{Ran}\left(B^{1 / 2}\right)$. As mentioned in the introduction, the infimum problem for a finite dimensional space $\mathcal{H}$ was solved in Ref. 19 by showing that $A \wedge B$ exists if and only if $A \wedge P_{A, B}$ and $B \wedge P_{A, B}$ are comparable, and that $A \wedge B$ is the smaller of $A \wedge P_{A, B}$ and $B \wedge P_{A, B}$. The following proposition shows that for $\operatorname{dim} \mathcal{H}=\infty$ the infimum problem for $A$ and $B$ can be reduced to the same problem for the "smaller" operators $A \wedge P_{A, B}$ and $B \wedge P_{A, B}$. In Sec. IV we will see that in this case the infimum problem cannot be solved in the same fashion, as conjectured in Ref. 19.

Proposition 2.4: Let $A, B \in \mathcal{E}(\mathcal{H})$. Then $A \wedge B$ exists if and only if $\left(A \wedge P_{A, B}\right) \wedge\left(B \wedge P_{A, B}\right)$ exists. In this case $A \wedge B=\left(A \wedge P_{A, B}\right) \wedge\left(B \wedge P_{A, B}\right)$.

Proof: Note first that the operators $A \wedge P_{A, B}$ and $B \wedge P_{A, B}$ exist, e.g., by Theorem 2.2.

Let us assume that $\left(A \wedge P_{A, B}\right) \wedge\left(B \wedge P_{A, B}\right)$ exists and let $C \in \mathcal{E}(\mathcal{H})$ be such that $C \leqslant A, B$, thus we have $\operatorname{Ran}\left(C^{1 / 2}\right) \subseteq \operatorname{Ran}\left(A^{1 / 2}\right) \cap \operatorname{Ran}\left(B^{1 / 2}\right) \subseteq \operatorname{Ran}\left(P_{A, B}\right)$ and hence $C \leqslant P_{A, B}$. Therefore, $C$ $\leqslant A \wedge P_{A, B}$ and $C \leqslant B \wedge P_{A, B}$ and hence, by the majorization theorem as in Ref. 6, $C$ $\leqslant\left(A \wedge P_{A, B}\right) \wedge\left(B \wedge P_{A, B}\right)$. Taking into account that $\left(A \wedge P_{A, B}\right) \wedge\left(B \wedge P_{A, B}\right) \leqslant A, B$ it follows that $A \wedge B$ exists and equals $\left(A \wedge P_{A, B}\right) \wedge\left(B \wedge P_{A, B}\right)$.

Conversely, let us assume that $A \wedge B$ exist. Then, $A \wedge B \leqslant P_{A, B}$. This relation and $A \wedge B \leqslant A, B$ give $A \wedge B \leqslant A \wedge P_{A, B}, A \wedge B \leqslant B \wedge P_{A, B}$. Let $C \in \mathcal{E}(\mathcal{H})$ be such that $C \leqslant A \wedge P_{A, B}, B \wedge P_{A, B}$. Then $C$ $\leqslant A, B, P_{A, B}$ and, in particular, $C \leqslant A \wedge B$.

One may ask for which orthogonal projections $P$ except $P_{A, B}$ the statement of Proposition 2.4 is true. It turns out that $P_{A, B}$ is the infimum of the set of those projections $P$.

Theorem 2.5: Let $A, B \in \mathcal{E}(\mathcal{H})$ such that $A \wedge B$ exists. Let $\Pi_{A, B}$ be the set of all orthogonal projections subject to the properties that $(A \wedge P) \wedge(B \wedge P)$ exists and $(A \wedge P) \wedge(B \wedge P)=A \wedge B$. Then

$$
\Pi_{A, B}=\left\{P \in \mathcal{P}(\mathcal{H}) \mid P_{A, B} \leqslant P\right\} .
$$

In order to prove the above stated proposition, we first consider the connection of parallel sum with the infimum of quantum effects (see also Ref. 2). To see this, instead of giving the original definition as in Ref. 8, we prefer to introduce the parallel sum of two quantum effects by means of the characterization of Pekarev-Shmulyan, ${ }^{20}$ 


$$
\langle(A: B) h, h\rangle=\inf \{\langle A a, a\rangle+\langle B b, b\rangle \mid h=a+b\}, \text { for all } h \in \mathcal{H} .
$$

Theorem 2.6: (Refs. 8 and 20) Let $A, B \in \mathcal{B}(\mathcal{H})^{+}$. Then

(i) $0 \leqslant A: B \leqslant A, B$,

(ii) $A: B=B: A$,

(iii) $\quad \operatorname{Ran}\left((A: B)^{1 / 2}\right)=\operatorname{Ran}\left(A^{1 / 2}\right) \cap \operatorname{Ran}\left(B^{1 / 2}\right)$,

(iv) if $A_{1}, B_{1} \in \mathcal{B}(\mathcal{H})^{+}$are such that $A \leqslant A_{1}$ and $B \leqslant B_{1}$, then $A: B \leqslant A_{1}: B_{1}$,

(v) if $A+B$ is boundedly invertible, then $\|A: B\|=A(A+B)^{-1} B$,

(vi) If $A_{n} \searrow A$ and $B_{n} \searrow B$ strongly, then $A_{n}: B_{n} \searrow A: B$ strongly.

In view of the properties of the parallel sum listed above, a moment of thought shows that if $P, Q \in \mathcal{P}(\mathcal{H})$, that is, $P$ and $Q$ are orthogonal projections in $\mathcal{H}$, then $P \wedge Q$ over $\mathcal{E}(\mathcal{H})$ always exists and coincides with the orthogonal projection onto $\operatorname{Ran}(P) \cap \operatorname{Ran}(Q)$. By Theorem 4.3 in Ref. 8 we also have $P \wedge Q=2(P: Q)$.

Lemma 2.7: Let $A, B \in \mathcal{E}(\mathcal{H})$ be such that $A \wedge B$ exists. Then

(i) $\quad \operatorname{Ran}\left((A \wedge B)^{1 / 2}\right)=\operatorname{Ran}\left((A: B)^{1 / 2}\right)$,

(ii) $\quad(A \wedge B)^{1 / 2}=(A: B)^{1 / 2} V$ for some boundedly invertible operator $V \in \mathcal{B}(\mathcal{H})$,

(iii) $A: B \leqslant A \wedge B \leqslant \gamma(A: B)$, for some $\gamma>0$.

Proof: Since $A \wedge B \leqslant A$ it follows that $\operatorname{Ran}\left((A \wedge B)^{1 / 2}\right) \subseteq \operatorname{Ran}\left(A^{1 / 2}\right)$. Similarly we have $\operatorname{Ran}\left((A \wedge B)^{1 / 2}\right) \subseteq \operatorname{Ran}\left(B^{1 / 2}\right)$, hence $\operatorname{Ran}\left((A \wedge B)^{1 / 2}\right) \subseteq \operatorname{Ran}\left(A^{1 / 2}\right) \cap \operatorname{Ran}\left(B^{1 / 2}\right)=\operatorname{Ran}\left((A: B)^{1 / 2}\right)$.

For the converse inclusion, note that $A: B \leqslant A$ and $A: B \leqslant B$; since $A: B \leqslant A: I=A(A+I)^{-1} \leqslant A$. Thus, by the definition of $A \wedge B$, it follows that $A: B \leqslant A \wedge B$. In particular, this proves that $\operatorname{Ran}\left((A \wedge B)^{1 / 2}\right) \supseteq \operatorname{Ran}\left((A: B)^{1 / 2}\right)$, and hence (i) is proved. 6.

The assertions (ii) and (iii) are consequences of (i) and the majorization theorem as in Ref.

Lemma 2.8: If $A, B \in \mathcal{E}(\mathcal{H})$ and $A \wedge B$ exists, then $A \wedge B \leqslant P_{A, B}$ and $\operatorname{Ran}(A \wedge B)$ is dense in $\operatorname{Ran}\left(P_{A, B}\right)$.

Proof: This is a consequence of Theorem 2.6 and Lemma 2.7.

We now come back to Theorem 2.5.

Proof of Theorem 2.5: Let $P \in \Pi_{A, B}$. Then $A \wedge B \leqslant P$ and hence $\overline{\operatorname{Ran}(A \wedge B)} \subseteq \operatorname{Ran}(P)$. Therefore, by Lemma $2.8 \operatorname{Ran}\left(P_{A, B}\right) \subseteq \operatorname{Ran}(P)$, that is, $P_{A, B} \leqslant P$.

Assume that $P \geqslant P_{A, B}$. We claim that then $(A \wedge P) \wedge(B \wedge P)$ exists and it coincides with $\left(A \wedge P_{A, B}\right) \wedge\left(B \wedge P_{A, B}\right)$. Evidently, $\left(A \wedge P_{A, B}\right) \wedge\left(B \wedge P_{A, B}\right) \leqslant A \wedge P, B \wedge P$. Let $C \in \mathcal{E}(\mathcal{H})$ with $C$ $\leqslant A \wedge P, B \wedge P$. Then $C \leqslant A \wedge B \leqslant P_{A, B}$ and hence,

$$
C \leqslant\left(A \wedge P_{A, B}\right) \wedge\left(B \wedge P_{A, B}\right) .
$$

Therefore, $(A \wedge P) \wedge(B \wedge P)$ exists and, by Proposition 2.4 it coincides with $A \wedge B$.

\section{INFIMUM OF A QUANTUM EFFECT AND ITS NEGATION}

The negation $A^{\prime}$ of an effect $A$ is defined to be the effect $A^{\prime}=I-A$. Physically, $A^{\prime}$ is the effect $A$ with its values 1 and 0 reversed. In the following we present a characterization of when $A \wedge A^{\prime}$ exists in $\mathcal{E}(\mathcal{H})$ in terms of the location of the spectrum of $A$. The theorem essentially coincides with the result of Ando (Ref. 2, Theorem 2), the difference consists on that we express the condition with the help of the spectrum of $A$ and the proof is based on the matrix representations as in Sec. II. There is also a similar characterization in Ref. 13.

Theorem 3.1: Let $A$ be a quantum effect on the Hilbert space $\mathcal{H}$. Then the following assertions are equivalent:

(i) $\quad A \wedge(I-A)$ exists,

(ii) $\quad \sigma(A)$, the spectrum of $A$, is contained either in $\{0\} \cup\left[\frac{1}{2}, 1\right]$ or in $\left[0, \frac{1}{2}\right] \cup\{1\}$,

(iii) $A \wedge P_{A, I-A}$ and $(I-A) \wedge P_{A, I-A}$ are comparable, that is, either $A \wedge P_{A, I-A} \leqslant(I-A) \wedge P_{A, I-A}$ or $(I-A) \wedge P_{A, I-A} \leqslant A \wedge P_{A, I-A}$. 
In addition, if either of the above holds, letting $g \in C([0,1])$ be the function

$$
g(t)=\min (t, 1-t)= \begin{cases}t, & 0 \leqslant t \leqslant \frac{1}{2}, \\ 1-t, & \frac{1}{2} \leqslant t \leqslant 1,\end{cases}
$$

we have, by continuous functional calculus, $A \wedge(I-A)=g(A)$.

Proof: Let $E_{A}$ denote the spectral function of $A$. In view of Proposition $2.4, A \wedge(I-A)$ exists if and only if $\left(A \wedge P_{A, I-A}\right) \wedge\left((I-A) \wedge P_{A, I-A}\right)$ exists. A moment of thought shows that $P_{A, I-A}$ $=E_{A}((0,1))$ and hence, by Remark 2.3, we have that $A \wedge P_{A, I-A}=A E_{A}((0,1))$ and $(I-A) \wedge P_{A, I-A}$ $=(I-A) E_{A}((0,1))$. Thus, without restricting the generality, we can and will assume in the following that 0 and 1 are not eigenvalues of $A$. Now, the equivalence of (ii) with (iii) is a matter of elementary spectral theory for selfadjoint operators, hence we will prove only the equivalence of (i) and (ii).

To prove that (ii) implies (i), let us assume that $\sigma(A)$ is contained either in $\{0\} \cup\left[\frac{1}{2}, 1\right]$ or in $\left[0, \frac{1}{2}\right] \cup\{1\}$. To make a choice, let us assume that $\sigma(A) \subseteq\{0\} \cup\left[\frac{1}{2}, 1\right]$. Since, by assumption, 0 is not an eigenvalue of $A$, it follows that $\sigma(A) \subseteq\left[\frac{1}{2}, 1\right]$. Then $A \geqslant I-A$ and clearly $A \wedge(I-A)=I-A$ $=g(A)$, where the function $g$ is defined as in (3.1). A similar argument holds in case we assume $\sigma(A) \subseteq\left[0, \frac{1}{2}\right] \cup\{1\}$; in this case $A \wedge(I-A)=A=g(A)$.

Conversely, let us assume that $A \wedge(I-A)=D$, the infimum of $A$ and $I-A$ over $\mathcal{E}(\mathcal{H})$, exists. Using the spectral measure $E_{A}$ of $A$, let $E_{1}=E_{A}\left([0,1 / 2], A_{1}=A \mid E_{1} \mathcal{H}, E_{2}=E_{A}((1 / 2,1]), A_{2}\right.$ $=A \mid E_{2} \mathcal{H}$. We write $D$ as an operator matrix with respect to the decomposition $\mathcal{H}=E_{1} \mathcal{H} \oplus E_{2} \mathcal{H}$,

$$
D=\left[\begin{array}{cc}
D_{1} & D_{1}^{1 / 2} \Gamma D_{2}^{1 / 2} \\
D_{2}^{1 / 2} \Gamma^{*} D_{1}^{1 / 2} & D_{2}
\end{array}\right],
$$

with contractive $\Gamma \in \mathcal{B}\left(E_{2} \mathcal{H}, E_{1} \mathcal{H}\right)$, cf. Theorem 2.1. Since $g(A) \leqslant A, I-A$, by the definition of $D$ we have

$$
0 \leqslant D-g(A)=\left[\begin{array}{cc}
D_{1}-A_{1} & D_{1}^{1 / 2} \Gamma D_{2}^{1 / 2} \\
D_{2}^{1 / 2} \Gamma^{*} D_{1}^{1 / 2} & D_{2}-\left(I_{2}-A_{2}\right)
\end{array}\right] .
$$

Therefore, $0 \leqslant D_{1}-A_{1}$ while taking into account that $D \leqslant A$ it follows that $D_{1} \leqslant A_{1}$, hence $D_{1}$ $=A_{1}$. Similarly, $0 \leqslant D_{2}-\left(I_{2}-A_{2}\right)$ and, since $D \leqslant I-A$ it follows $D_{2} \leqslant I_{2}-A_{2}$, hence $D_{2}=I_{2}-A_{2}$. Thus, the main diagonal of the matrix in (3.2) is null, hence (e.g., by Theorem 2.1) it follows that $D=g(A)$.

Further, let $\varepsilon \in(0,1 / 4)$, and consider the operators

$$
E_{\varepsilon, 1}=E_{A}((\varepsilon,-\varepsilon+1 / 2)), \quad E_{\varepsilon, 2}=E_{A}((\varepsilon+1 / 2,1-\varepsilon)) .
$$

Denote $E_{\varepsilon}=E_{\varepsilon, 1}+E_{\varepsilon, 2}$ and $A_{\varepsilon}=A \mid E_{\varepsilon} \mathcal{H}$. We show that $A_{\varepsilon} \wedge\left(I-A_{\varepsilon}\right)$ exists. To see this, we remark that, as proven before, $g(A)=A \wedge(I-A)$, so we actually show that $D_{\varepsilon}=D \mid E_{\varepsilon} \mathcal{H}=g\left(A_{\varepsilon}\right)$ coincides with $A_{\varepsilon} \wedge\left(I-A_{\varepsilon}\right)$. Indeed, assume that for some $C_{\varepsilon} \in \mathcal{E}\left(E_{\varepsilon} \mathcal{H}\right)$ we have $C_{\varepsilon} \leqslant A_{\varepsilon}, I-A_{\varepsilon}$. Then, letting $C=C_{\varepsilon} E_{\varepsilon} \in \mathcal{E}(\mathcal{H})$ it follows that $C \leqslant A, I-A$. Since $D=A \wedge(I-A)$ this implies $C \leqslant D$ and hence $C_{\varepsilon} \leqslant D_{\varepsilon}$. Therefore, $D_{\varepsilon}$ coincides with $A_{\varepsilon} \wedge\left(I-A_{\varepsilon}\right)$.

We finally prove that (i) implies (ii). Assume that (i) holds and (ii) is not true. Then there exists $\varepsilon \in(0,1 / 4)$ such that $E_{\varepsilon, 1} \neq 0$ and $E_{\varepsilon, 2} \neq 0$, where we use the notation as in (3.3). Letting

$$
A_{\varepsilon, 1}=A\left|E_{\varepsilon, 1} \mathcal{H}, \quad A_{\varepsilon, 2}=A\right| E_{\varepsilon, 2} \mathcal{H},
$$

and $d=\varepsilon(1+\sqrt{3})^{-1}$, consider an arbitrary contraction $T \in \mathcal{B}\left(E_{\varepsilon, 2} \mathcal{H}, E_{\varepsilon, 1} \mathcal{H}\right)$. In the following all operator matrices are understood with respect to the decomposition $E_{\varepsilon, 1} \mathcal{H} \oplus E_{\varepsilon, 2} \mathcal{H}$. Then, letting 


$$
\begin{aligned}
C & =\left[\begin{array}{ll}
A_{\varepsilon, 1}-\mathrm{d} I_{\varepsilon, 1} & \sqrt{3} \mathrm{~d} T \\
\sqrt{3} \mathrm{~d} T^{*} & I_{\varepsilon, 2}-A_{\varepsilon, 2}-\mathrm{d} I_{\varepsilon, 2}
\end{array}\right]=\left[\begin{array}{ll}
A_{\varepsilon, 1}-\varepsilon I_{\varepsilon, 1}+\sqrt{3} \mathrm{~d} I_{\varepsilon, 1} & \sqrt{3} \mathrm{~d} T \\
\sqrt{3} \mathrm{~d} T^{*} & I_{\varepsilon, 2}-A_{\varepsilon, 2}-\varepsilon I_{\varepsilon, 2}+\sqrt{3} \mathrm{~d} I_{\varepsilon, 2}
\end{array}\right] \\
& =\left[\begin{array}{ll}
A_{\varepsilon, 1}-\varepsilon I_{\varepsilon, 1} & 0 \\
0 & I_{\varepsilon, 2}-A_{\varepsilon, 2}-\varepsilon I_{\varepsilon, 2}
\end{array}\right]+\sqrt{3} d\left[\begin{array}{ll}
I_{\varepsilon, 1} & T \\
T^{*} & I_{\varepsilon, 2}
\end{array}\right] \geqslant 0,
\end{aligned}
$$

we have

$$
A_{\varepsilon}-C=\left[\begin{array}{ll}
\mathrm{d} I_{\varepsilon, 1} & -\sqrt{3} \mathrm{~d} T \\
-\sqrt{3} \mathrm{~d} T^{*} & 2 A_{\varepsilon, 2}-I_{\varepsilon, 2}+\mathrm{d} I_{\varepsilon, 2}
\end{array}\right]=\left[\begin{array}{ll}
0 & 0 \\
0 & 2 A_{\varepsilon, 2}-I_{\varepsilon, 2}-2 \mathrm{~d} I_{\varepsilon, 2}
\end{array}\right]+d\left[\begin{array}{ll}
I_{\varepsilon, 1} & -\sqrt{3} T \\
-\sqrt{3} T^{*} & 3 I_{\varepsilon, 2}
\end{array}\right] \geqslant 0
$$

and

$$
\begin{aligned}
I-A_{\varepsilon}-C & =\left[\begin{array}{ll}
I_{\varepsilon, 1}-2 A_{\varepsilon, 1}+\mathrm{d} I_{\varepsilon, 1} & -\sqrt{3} T \\
-\sqrt{3} \mathrm{~d} T^{*} & \mathrm{~d} I_{\varepsilon, 2}
\end{array}\right]=\left[\begin{array}{ll}
I_{\varepsilon, 1}-2 A_{\varepsilon, 1}-2 \mathrm{~d} I_{\varepsilon, 1} & 0 \\
0 & 0
\end{array}\right]+d\left[\begin{array}{ll}
3 I_{\varepsilon, 1} & -\sqrt{3} T \\
-\sqrt{3} \mathrm{~d} T^{*} & I_{\varepsilon, 2}
\end{array}\right] \\
& \geqslant 0 .
\end{aligned}
$$

But, the operator

$$
\left(A_{\varepsilon} \wedge\left(I_{\varepsilon}-A_{\varepsilon}\right)\right)-C=g\left(A_{\varepsilon}\right)-C=d\left[\begin{array}{ll}
I_{\varepsilon, 1} & -\sqrt{3} T \\
-\sqrt{3} T^{*} & I_{\varepsilon, 2}
\end{array}\right]
$$

is not non-negative for some choices of $T$, unless at least one of the spectral projections $E_{\varepsilon, 1}$ and $E_{\varepsilon, 2}$ is trivial. Since $\varepsilon$ is arbitrarily small, it follows that $A$ cannot simultaneously have spectral points in $(0,1 / 2)$ and $(1 / 2,1)$. Therefore, (i) implies (ii).

\section{TWO EXAMPLES}

In this section we answer in the negative a question raised in Ref. 19. First we recall how the problem of the existence of the infimum of $A$ and $B$ in $\mathcal{E}(\mathcal{H})$ can be reduced to the infimum problem for some quantum effects and their negations. Assume, in addition, that $\operatorname{Ker}(A+B)=0$. Let $f_{A+B}$ be the affine (that is, linear on convex combinations) mapping defined as in Ref. 9 by

$$
f_{A+B}:\{C \mid 0 \leqslant C \leqslant A+B\} \rightarrow\left\{D \mid 0 \leqslant D \leqslant P_{A+B}\right\},
$$

with $C=(A+B)^{1 / 2} f_{A+B}(C)(A+B)^{1 / 2}$. By Theorem 2.2 in Ref. 9, $f_{A+B}$ is well defined. Since $f_{A+B}$ is an affine isomorphism, $A \wedge B$ exists if and only if $f_{A+B}(A) \wedge f_{A+B}(B)$ exists. As

$$
f_{A+B}(A)+f_{A+B}(B)=f_{A+B}(A+B)=I
$$

we are in the situation of Theorem 3.1.

Actually, the following more general fact holds.

Lemma 4.1: Let $A \in \mathcal{E}(\mathcal{H}), 0 \leqslant C, D \leqslant A$, and consider the mapping $f_{A}$ as defined in (4.1). Then $C \wedge D$ exists if and only if $f_{A}(C) \wedge f_{A}(D)$ exists and, in this case, we have

$$
f_{A}(C \wedge D)=f_{A}(C) \wedge f_{A}(D) .
$$

Proof: This is a consequence of Theorem 2.5 in Ref. 9.

By Proposition 2.4, the infimum of $A$ and $B$ exists if and only if the infimum of $A \wedge P_{A, B}$ and $B \wedge P_{A, B}$ exists or, equivalently, the infimum of the restrictions $\tilde{A}:=A \wedge P_{A, B} \mid P_{A, B} \mathcal{H}$ and $\widetilde{B}$ $:=B \wedge P_{A, B} \mid P_{A, B} \mathcal{H}$ exists. Since $\operatorname{Ker}(\tilde{A}+\widetilde{B})=\{0\}, \widetilde{A} \wedge \widetilde{B}$ exists if and only if $f_{\tilde{A}+\tilde{B}}(\widetilde{A}) \wedge f_{\tilde{A}+\tilde{B}}(\widetilde{B})$ exists, and for the pair $f_{\tilde{A}+\tilde{B}}(\widetilde{A}), f_{\tilde{A}+\tilde{B}}(\widetilde{B})$ we observe that Theorem 3.1 applies. Therefore, under the additional assumptions that 0 and 1 are not eigenvalues of $f_{\tilde{A}+\tilde{B}}(\widetilde{A})$ and $f_{\tilde{A}+\tilde{B}}(\widetilde{B}), \widetilde{A} \wedge \widetilde{B}$ exists if and only if $A \wedge P_{A, B}$ and $B \wedge P_{A, B}$ are comparable; in this case, $A \wedge B$ coincides with the smaller of the 
$A \wedge P_{A, B}$ and $B \wedge P_{A, B}$. For a finite dimensional Hilbert space it was proven in Ref. 19 that the infimum of the operators $A \wedge P_{A, B}$ and $B \wedge P_{A, B}$ exists if and only if they are comparable.

The next example shows that, contrary to the finite dimensional case, we may have two quantum effects $B_{1}$ and $B_{2}$ for which $B_{1} \wedge B_{2}$ exists, but $\left(B_{1} \wedge P_{B_{1}, B_{2}}\right)$ and $\left(B_{2} \wedge P_{B_{1}, B_{2}}\right)$ are not comparable.

Example 4.2: Let $\mathcal{H}=L^{2}[-1,1]$ and $A$ be the operator of multiplication with the square of the independent variable on $\mathcal{H},(A x)(t)=t^{2} x(t)$, for all $x \in L^{2}[-1,1]$. Then $A$ is a non-negative contraction on $\mathcal{H}$, that is, a quantum effect, and the same is its square root $A^{1 / 2}$, that is, $\left(A^{1 / 2} x\right)(t)$ $=|t| x(t), x \in L^{2}[-1,1]$. Note that $A$, and hence $A^{1 / 2}$, are injective.

Let 1 be the constant function equal to 1 on $[-1,1], \theta(t):=\operatorname{sgn}(t)$, and $\chi_{ \pm}:=\frac{1}{2}(\mathbf{1} \pm \theta)$, the characteristic functions of $[0,1]$ and, respectively, $[-1,0]$. All these functions are in $L^{2}[-1,1]$. Note that 1 and $\theta$ span the same two dimensional space as $\chi_{ \pm}$. Denote

$$
\mathcal{H}_{0}=\mathcal{H} \ominus \operatorname{span}\{\mathbf{1}, \theta\}=\mathcal{H} \ominus \operatorname{span}\left\{\chi_{+}, \chi_{-}\right\} .
$$

With respect to the decomposition

$$
\mathcal{H}=\mathrm{C} \mathbf{1} \oplus \mathrm{C} \theta \oplus \mathcal{H}_{0}
$$

consider two quantum effects $C_{1}$ and $C_{2}$ on $\mathcal{H}$ defined by

$$
C_{1}=\left[\begin{array}{ccc}
0 & 0 & 0 \\
0 & 1 & 0 \\
0 & 0 & \frac{1}{2} I_{0}
\end{array}\right], \quad C_{1}=\left[\begin{array}{ccc}
1 & 0 & 0 \\
0 & 0 & 0 \\
0 & 0 & \frac{1}{2} I_{0}
\end{array}\right],
$$

where $I_{0}$ is the identity operator on $\mathcal{H}_{0}$. Clearly we have $C_{1}+C_{2}=I$ and letting

$$
B_{1}=A^{1 / 2} C_{1} A^{1 / 2}, \quad B_{2}=A^{1 / 2} C_{2} A^{1 / 2},
$$

we have

$$
B_{1}+B_{2}=A \text {. }
$$

Comparing the spectra of $C_{1}$ and $C_{2}$ and using Theorem 3.1, it follows that $C_{1} \wedge C_{2}$ exists, but $C_{1}$ and $C_{2}$ are not comparable. Therefore, using Lemma 4.1, it follows that $B_{1} \wedge B_{2}$ exists, but $B_{1}$ and $B_{2}$ are not comparable. In the following we will prove that $P_{B_{1}, B_{2}}=I$, that is, $\operatorname{Ran}\left(B_{1}^{1 / 2}\right) \cap \operatorname{Ran}\left(B_{2}^{1 / 2}\right)$ is dense in $\mathcal{H}$. We divide the proof in several steps.

Step 1: $A^{1 / 2} \mathcal{H}_{0}$ is dense in $\mathcal{H}$.

Indeed, let $f \in \mathcal{H}=L^{2}[-1,1]$ be a function such that for all $h_{0} \in \mathcal{H}_{0}$ we have

$$
0=\left\langle A^{1 / 2} h_{0}, f\right\rangle=\left\langle h_{0}, A^{1 / 2} f\right\rangle .
$$

Then $A^{1 / 2} f$ is a linear combination of the functions $\mathbf{1}$ and $\theta$, that is, there exist scalars $\alpha$ and $\beta$ such that

$$
|t| f(t)=\alpha+\beta \operatorname{sgn}(t), \quad t \in[-1,1]
$$

and hence

$$
f(t)=\frac{\alpha+\beta \operatorname{sgn}(t)}{|t|}= \begin{cases}\frac{\alpha+\beta}{t}, & 0<t \leqslant 1, \\ \frac{\beta-\alpha}{t}, & -1 \leqslant t<0 .\end{cases}
$$

Since $f \in L^{2}[-1,1]$ this shows that $f=0$ and the claim is proven.

Let us consider the following linear manifolds in $\mathcal{H}$ : 


$$
\begin{gathered}
\mathcal{F}:=\left\{f \in L^{2}[-1,1] \mid f \text { piecewise constant }\right\}, \\
\mathcal{F}_{0}:=\left\{f \in \mathcal{F} \mid \exists \varepsilon>0 \text { s.t. } f \mid(-\varepsilon, \varepsilon)=0,\left\langle f, \chi_{-}\right\rangle=\left\langle f, \chi_{+}\right\rangle=0\right\} .
\end{gathered}
$$

Step 2: $\mathcal{F}_{0}$ is dense in $\mathcal{H}_{0}$.

Indeed, to see this, let us first note that $\mathcal{F}_{0} \subset \mathcal{H}_{0}$. If $h_{0}$ is an arbitrary vector in $\mathcal{H}_{0}$ and $\varepsilon$ $>0$, there exists $f_{1} \in \mathcal{F}$ such that

$$
\left\|h_{0}-f_{1}\right\| \leqslant \frac{\varepsilon}{8} \text { hence }\left|\left\langle h_{0}-f_{1}, \chi_{ \pm}\right\rangle\right| \leqslant \frac{\varepsilon}{8} .
$$

Moreover, there exists $f_{2} \in \mathcal{F}$ such that it is zero in a neighbourhood of zero and

$$
\left\|f_{1}-f_{2}\right\| \leqslant \frac{\varepsilon}{8}
$$

Consequently,

$$
\left\|h_{0}-f_{2}\right\| \leqslant \frac{\varepsilon}{4} \quad \text { and hence }\left|\left\langle h_{0}-f_{2}, \chi_{ \pm}\right\rangle\right| \leqslant \frac{\varepsilon}{4} \text {. }
$$

Let

$$
f_{3}=f_{2}+2 \chi_{[1 / 2,1]}\left\langle h_{0}-f_{2}, \chi_{+}\right\rangle+2 \chi_{[-1,-1 / 2]}\left\langle h_{0}-f_{2}, \chi_{-}\right\rangle .
$$

Then, from the choice of $f_{2}$ it follows

$$
\left\langle f_{3}, \chi_{+}\right\rangle=\left\langle f_{2}, \chi_{+}\right\rangle+\left\langle h_{0}-f_{2}, \chi_{+}\right\rangle=\left\langle h_{0}, \chi_{+}\right\rangle=0
$$

and

$$
\left\langle f_{3}, \chi_{-}\right\rangle=\left\langle f_{2}, \chi_{-}\right\rangle+\left\langle h_{0}-f_{2}, \chi_{-}\right\rangle=\left\langle h_{0}, \chi_{-}\right\rangle=0,
$$

hence $f_{3} \in \mathcal{F}_{0}$. Finally, from (4.2), (4.3), and (4.4) we get

$$
\left\|h_{0}-f_{3}\right\| \leqslant\left\|h_{0}-f_{1}\right\|+\left\|f_{1}-f_{2}\right\|+\left\|f_{2}-f_{3}\right\| \leqslant \varepsilon,
$$

and the claim is proven.

Finally, we prove the following.

Step 3: $P_{B_{1}, B_{2}}=I$, that is, $\operatorname{Ran}\left(B_{1}^{1 / 2}\right) \cap \operatorname{Ran}\left(B_{2}^{1 / 2}\right)$ is dense in $\mathcal{H}$.

In the following we are using the inverse operator $A^{-1 / 2}$ on its range. By the preceding claim, $A^{1 / 2}\left(A^{-1 / 2} \mathcal{F}_{0}\right)$ is a linear submanifold in $\mathcal{H}_{0}$ and dense in it. Since the restrictions of $C_{1}$ and $C_{2}$ to $\mathcal{H}_{0}$ coincide with $\frac{1}{2} I_{0}$, it follows that the linear manifolds $C_{1} A^{1 / 2}\left(A^{-1} \mathcal{F}_{0}\right)$ and $C_{2} A^{1 / 2}\left(A^{-1} \mathcal{F}_{0}\right)$ coincide and are dense in $\mathcal{H}_{0}$. Consequently, the linear manifolds $A^{1 / 2} C_{1} A^{1 / 2}\left(A^{-1} \mathcal{F}_{0}\right)$ and $A^{1 / 2} C_{2} A^{1 / 2}\left(A^{-1} \mathcal{F}_{0}\right)$ coincide and, by Step 1 and Step 2, they are dense in $\mathcal{H}$. Thus, the linear manifold,

$$
\mathcal{L}=B_{1}\left(A^{-1 / 2} \mathcal{F}_{0}\right)=B_{2}\left(A^{-1 / 2} \mathcal{F}_{0}\right) \subseteq \operatorname{Ran}\left(B_{1}\right) \cap \operatorname{Ran}\left(B_{2}\right) \subseteq \operatorname{Ran}\left(B_{1}^{1 / 2}\right) \cap \operatorname{Ran}\left(B_{2}^{1 / 2}\right),
$$

is dense in $\mathcal{H}$. This concludes the proof of the last step, and the example.

In order to explain the connection with the characterization of the existence of infimum obtained by Ando in Ref. 2 we consider the comparison of $A \wedge P_{A, B}$ with the generalized shorted operator, as considered in Ref. 2.

Lemma 4.3: Let $A, B \in \mathcal{E}(\mathcal{H})$. Then, for any sequence $\alpha_{n}$ of positive numbers that converge increasingly to infinity, we have

$$
\text { SO- } \lim _{n \rightarrow \infty}\left(A: \alpha_{n} B\right) \leqslant A \wedge P_{A, B},
$$


and the limit does not depend on the sequence $\left(\alpha_{n}\right)$.

Proof: First note that the sequence of positive operators $A: \alpha_{n} B$ is nondecreasing and bounded by $A$, cf. Ref. 8. Consequently, the strong operator limit exists and does not depend on the sequence $\alpha_{n}$ increasing to infinity. We thus can take $\alpha_{n}=n$. Since the parallel sum is strongly continuous in the second variable with respect to nondecreasing sequences, cf. Theorem 2.6, we have $A: n B \leqslant A$ and, since $\operatorname{Ran}\left((A: n B)^{1 / 2}\right)=\operatorname{Ran}\left(A^{1 / 2}\right) \cap \operatorname{Ran}\left(B^{1 / 2}\right)$ it follows $A: n B \leqslant P_{A, B}$ and hence (4.5) holds.

Given two positive operators $A$ and $B$, the generalized shorted operator $[B] A$ is defined (see Ref. 1) by

$$
[B] A=\lim _{n \rightarrow \infty} A:(n B) .
$$

The main result in Ref. 2 states that the infimum $A \wedge B$ exists if and only $[B] A$ and $[A] B$ are comparable and, in this case, $A \wedge B$ is the smaller of $[A] B$ and $[B] A$. In view of this result and our Example 4.2, it follows that, in general, (4.5) cannot be improved to equality. Here we have a simpler example emphasizing this fact.

Example 4.4: Let $\mathcal{H}=L^{2}[0,1]$ and $A$ the operator of multiplication with the function $t^{2}$. Then $A$ is bounded, contractive, and positive. In addition, $A^{1 / 2}$ is the operator of multiplication with the independent variable $t$. Note that both $A$ and $A^{1 / 2}$ are injective.

Further, let 1 be the function constant 1 in $L^{2}[0,1]$ and note that it does not belong to the range of either $A$ or $A^{1 / 2}$. Let $C$ be a non-negative contraction in $\mathcal{H}$ with kernel $C 1$ and define $B=A^{1 / 2} C A^{1 / 2}$. Then the operator $B$ is injective and hence its range is dense in $\mathcal{H}$. Since $\operatorname{Ran}(B) \subseteq \operatorname{Ran}\left(B^{1 / 2}\right)$ and, by construction, $\operatorname{Ran}(B) \subseteq \operatorname{Ran}\left(A^{1 / 2}\right)$ as well, it follows that $\operatorname{Ran}\left(A^{1 / 2}\right) \cap \operatorname{Ran}\left(B^{1 / 2}\right)$ is dense in $\mathcal{H}$, hence $P_{A, B}=I$.

For each $n \geqslant 1$ consider the function $v_{n} \in L^{2}[0,1]$ defined by

$$
v_{n}(t)=\left\{\begin{array}{lc}
0, & 0 \leqslant t \leqslant 1 / n, \\
1 / t & 1 / n<t \leqslant 1 .
\end{array}\right.
$$

Note that $A^{1 / 2} v_{n}=\chi_{(1 / n, 1]}$, the characteristic function of the interval $(1 / n, t]$. Taking into account that the sequence of functions $\chi_{(1 / n, 1]}$ converges in norm to the function $\mathbf{1}$, it follows that

$$
\left\langle B v_{n}, v_{n}\right\rangle=\left\langle C A^{1 / 2} v_{n}, A^{1 / 2} v_{n}\right\rangle=\left\langle C \chi_{(1 / n, 1]}, \chi_{(1 / n, 1]}\right\rangle \rightarrow\langle C \mathbf{1}, \mathbf{1}\rangle=0 .
$$

Let $\alpha_{n}$ be a sequence of positive numbers increasing to $+\infty$ and such that $\alpha_{n}\left\langle B v_{n}, B v_{n}\right\rangle$ converges to 0 . It is easy to see that this is always possible. Then using the characterization of the parallel sum as in Theorem 2.6.(vi), for arbitrary $n \geqslant m>2$ we have

$$
\begin{aligned}
\left\langle\left(A: \alpha_{n} B\right) v_{m} v_{m}\right\rangle= & \inf \left\{\langle A u, u\rangle+\alpha_{n}\langle B v, v\rangle \mid v_{m}=u+v\right\}=\inf \left\{\left\langle A\left(v_{m}-v\right), v_{m}-v\right\rangle+\alpha_{n}\langle B v, v\rangle \mid v \in \mathcal{H}\right\} \\
= & \inf \left\{\left\langle A v_{m}, v_{m}\right\rangle-2 \operatorname{Re}\left\langle A v_{m}, v\right\rangle+\langle A v, v\rangle+\alpha_{n}\langle B v, v\rangle \mid v \in \mathcal{H}\right\} \leqslant\left\langle A v_{m}, v_{m}\right\rangle \\
& -2 \operatorname{Re}\left\langle A v_{m}, v_{n}\right\rangle+\left\langle A v_{n}, v_{n}\right\rangle+\alpha_{n}\left\langle B v_{n}, v_{n}\right\rangle=1-\frac{1}{m}-2+\frac{2}{m}+1-\frac{1}{n}+\alpha_{n}\left\langle B v_{n}, v_{n}\right\rangle \\
= & \frac{1}{m}-\frac{1}{n}+\alpha_{n}\left\langle B v_{n}, v_{n}\right\rangle \rightarrow \frac{1}{m}<\frac{1}{2} \text { as } n \rightarrow \infty .
\end{aligned}
$$

On the other hand,

$$
\left\langle A v_{m}, A v_{m}\right\rangle=1-\frac{1}{m} \geqslant \frac{1}{2} .
$$

Hence, we have strict inequality in (4.5). 
${ }^{1}$ Ando, T., "Lebesgue-type decomposition of positive operators," Acta Sci. Math., Széged 38, 253-260 (1976).

${ }^{2}$ Ando, T., "Problem of infimum in the positive cone," Analytic and Geometric Inequalities and Applications, Math. Appl. 478 (Kluwer Academic, Dordrecht, 1999).

${ }^{3}$ Busch, P., Grabowski, M., and Lahti, P. J., Operational Quantum Physics (Springer, Berlin, 1995).

${ }^{4}$ Busch, P., Lahti, P. J., and Middlestaedt, P., The Quantum Theory of Measurements (Springer, Berlin 1991).

${ }^{5}$ Davies, E. B., Quantum Theory of Open Systems (Academic, New York, 1976).

${ }^{6}$ Douglas, R. G., "On majorization, factorization, and range inclusion of operators on Hilbert space," Proc. Am. Math. Soc. 17, 413-415 (1966)

${ }^{7}$ Dvurečenskij, A. and Pulmannová, S., New Trends in Quantum Structures, Mathematics and its Applications, Vol. 516 (Kluwer Academic, Dordrecht, Ister Science, Bratislava 2000).

${ }^{8}$ Fillmore, P. A. and Williams, J. P., “On operator ranges,” Adv. Math. 7, 254-281 (1971).

${ }^{9}$ Gheondea, A. and Gudder. S., "Sequential product of quantum effects," Proc. Am. Math. Soc. 132, 503-512 (2004).

${ }^{10}$ Gudder, S., "Examples, problems and results in effect algebras," Int. J. Theor. Phys. 35, 2365-2376 (1996).

${ }^{11}$ Gudder, S., "Lattice properties of quantum effects," J. Math. Phys. 37, 2637-2642 (1996).

${ }^{12}$ Gudder, S. and Greechie, R., "Effect algebra counterexamples," Math. Slovaca 46, 317-325 (1996).

${ }^{13}$ Heinonen, T., Lahti, P., Pellonpää, J.-P, Pulmannová, S., and Ylinen, K., "The norm-1-property of a quantum observable," J. Math. Phys. 44, 1998-2008 (2003).

${ }^{14}$ Holevo, A. S., Probabilistic and Statistical Aspects of Quantum Theory (North-Holland, Amsterdam, 1982).

${ }^{15}$ Kadison, R., "Order properties of bounded self-adjoint operators," Proc. Am. Math. Soc. 34, 505-510 (1951).

${ }^{16}$ Kraus, K., States, Effects and Operations (Springer, Berlin, 1983).

${ }^{17}$ Lahti, P. J. and Maczynski, M., "On the order structure of the set of effects in quantum mechanics," J. Math. Phys. 36, 1673-1680 (1995).

${ }^{18}$ Ludwig, G., Foundations of Quantum Mechanics (Springer, Berlin 1983/1985), Vols. I and II.

${ }^{19}$ Moreland, T. and Gudder, S., "Infima of Hilbert space effects," Linear Algebr. Appl. 286, 1-17 (1999).

${ }^{20}$ Pekarev, E. L. and Shmulyan, Yu. L., "Parallel addition and parallel subtraction of operators," Izv. Akad. Nauk SSSR, Ser. Mat. 40, 366-387 (1976).

${ }^{21}$ Shmulyan, Yu. L., "An operator Hellinger integral," Mat. Sb. 49, 381-430 (1959). 